\title{
Avaliação da Capacidade Antimicrobiana de Nanopartículas de Prata Sintetizadas com Mel de Abelha
}

\section{Evaluation of the Antimicrobial Capacity of Silver Nanoparticles Synthesized with Bee Honey}

\author{
Gabriela Sousa Dourado ${ }^{\mathrm{a}}$ J Julio César Amaral Cardoso ${ }^{\mathrm{a}}$; Igor Feijão Cardoso ; Alan Kelbis Oliveira Lima ${ }^{\mathrm{b}}$; Silvia \\ Katrine Rabelo da Silva ${ }^{a}$; Arthur Abinader Vasconcelos ${ }^{\text {a; }}$ José Jeosafá V. Sousa Júnior ${ }^{\text {a; }}$ Paulo Sérgio Taube Junior*a \\ ${ }^{a}$ Instituto de Biodiversidade e Florestas, Universidade Federal do Oeste do Pará. PA, Brazil. \\ bUniversidade de Brasília, Programa de Pós-Graduação Stricto Sensu em Nanociência e Nanobiotecnologia. DF, Brazil. \\ *E-mail: pstjunior@yahoo.com.br
}

\begin{abstract}
Resumo
Nos últimos anos, as nanopartículas de prata (AgNPs) têm atraído muita atenção devido às suas largas aplicações em diferentes campos como biotecnologia, microeletrônica, armazenamento de informação óptica, medicina e conversão de energia. O presente trabalho teve como objetivo sintetizar nanopartículas de prata a partir de amostras de mel de abelhas Apis mellifera produzidos na cidade de Santarém-Pará, Brasil. Além disso, avaliar a atividade antimicrobiana dessas amostras, obtidas em dois períodos distintos (seco e chuvoso), frente a patógenos de interesse clínico: Staphylococcus aureus, Staphylococus epidermidis e Cândida krusei. Todas as amostras apresentaram estabilidade, homogeinidade e diâmetro hidrodinâmico médio de $600 \mathrm{~nm}$, tendo uma grande área superficial. Apresentaram índice de polidispersividade (PdI) médio de 0,36 e 0,28 , utilizando méis do período seco e chuvoso, respectivamente. O potencial Zeta foi negativo para 5 amostras do período seco. As linhagens de $S$. aureus, S. epidermides e C. krusei foram sensíveis à todas as AgNPs sintetizadas, sendo que as CMI para $S$. aureus e $S$. epidermides variaram de 0,17 a $10,79 \mathrm{mg} \mathrm{mL}^{-1}$ e para $C$. krusei de 0,08 a $10,79 \mathrm{mg} \mathrm{mL}^{-1}$. De acordo com estudos as AgNPs sintetizadas se mostram mais ativas em bactérias Gram negativas do que Gram positivo. No entanto, os elevados valores de CMI obtidos neste trabalho estão associados aos maiores tamanhos das nanoparticulas sintetizadas.
\end{abstract}

Palavras-chave: Apis melifera. Staphylococcus aureus. Staphylococus epidermidis. Candida krusei.

\begin{abstract}
In recent years, silver nanoparticles (AgNPs) have attracted a lot of attention due to their wide applications in different fields such as biotechnology, microelectronics, optical information storage, medicine, and energy conversion. The present work aimed to synthesize silver nanoparticles from honey samples from Apis mellifera bees produced in the Santarém-Pará, Brazil. In addition, to evaluate the antimicrobial activity of these samples, obtained in two distinct periods (dry and rainy), against pathogens of clinical interest: Staphylococcus aureus, Staphylococus epidermidis and Candida krusei. All samples showed stability, homogeneity and an average hydrodynamic diameter of 600 $\mathrm{nm}$, with a large surface area. They had an average polydispersity index (PdI) of 0.36 and 0.28 , using honeys from the dry and rainy periods, respectively. The Zeta potential was negative for 5 samples from the dry period. The strains of S. aureus, S. epidermides and C. krusei were sensitive to all synthesized AgNPs, with the MIC for S. aureus and S. epidermides ranging from 0.17 to $10.79 \mathrm{mg} \mathrm{mL}^{-1}$ and for C krusei from 0.08 to $10.79 \mathrm{mg} \mathrm{mL}^{-1}$. According to studies, the synthesized AgNPs are more active in Gram negative bacteria than Gram positive. However, the high MIC values obtained in this work are associated with the larger sizes of the synthesized nanoparticles.
\end{abstract}

Keywords: Apis mellifera. Staphylococcus aureus. Staphylococus epidermidis. Candida krusei.

\section{Introdução}

O mel é um fluído viscoso, aromático e doce produzido por abelhas melíferas a partir do néctar de flores ou mesmo através das excreções de insetos sugadores de plantas (BANDEIRA et al., 2018). Sua composição e constituída predominantemente por açúcares (e.g. glicose e frutose) e água, além de pequenas contribuições de vitaminas, enzimas minerais e compostos fenólicos (BALL, 2007; SONG; KIM, 2009). Nesse sentido além de ser um alimento natural rico em energia, o mel possuí inúmeros efeitos terapêuticos (digestivo e diurético) e medicinais (antibacteriano, antinflamatório, analgésico, expectorante, entre outros) (MEIRELES; CANÇADO, 2016; LAVINAS et al., 2019).

As nanopartículas (NPs) são aglomerados de átomos com tamanho na ordem de 1 a $100 \mathrm{~nm}$. Sendo que as nanopartículas metálicas apresentam boas propriedades antibacterianas e antifúngicas em relação aos compostos bioativos isolados. Nesse sentido, devido a crescente resistência dos microrganismos frente aos íons metálicos e antibióticos, cada vez mais pesquisas vêm sendo executadas para obtenção de NPs com maiores potenciais antimicrobianos (RAI et al., 2009).

Dentre estas NPs, as nanopartículas de prata (AgNPs) possuem alta eficiência contra doenças causadas por bactérias (Staphylococcus aureus, Escherichia coli e Pseudomonas aeruginoa) e fungos que causam infecções de pele (Candida albicans). Essas nanoparticulas apresentam melhores atividades quando comparadas ao uso isolado de compostos bioativos (RAI, 2013). Por exemplo, alguns trabalhos utilizando nanopartículas de prata sintetizadas a partir de mel apresentaram excelentes ações antimicrobianas (BALASOORIYA et al., 2017; HOSNY et al., 2017). 
Balasooriya et al. (2017) encontrou significativa atividade antimicrobiana contra uma ampla gama de linhagens bacterianas e fúngicas, sendo muito eficiente para inibição de Staphylococcus aureus. Já Hosny et al. ( 2017) observou que as AgNPs sintetizadas foram mais ativas contra as bactérias Gram-negativas testadas do que as Gram-positivas.

Vários métodos foram introduzidos para sintetizar e estabilizar AgNPs, entre os mais utilizados estão: redução química ou físico-química e técnicas eletroquímicas (HOSNY et al., 2017). Entretanto esses métodos possuem custos elevados, consomem muita energia e utilizam, normalmente, reagentes nocivos para o meio ambiente (GONZÁLEZ FÁ et al., 2017). A síntese verde de AgNPs a partir de produtos e matrizes naturais realizada em ultrassom é uma técnica que possui muitas vantagens sobre outros métodos de biossíntese, incluindo a prevenção de processos elaborados, como a manutenção de culturas celulares e/ou secagem e extração de materiais vegetais (VENU et al., 2011; OSKUEE et al., 2016).

Nesse contexto, uma vez que o mel apresenta em sua composição compostos capazes de reduzir e estabilizar a prata, além de ter uma atividade antimicrobiana natural, o mesmo tem grande potencial para ser empregado na síntese de AgNPs benéficas a saúde humana (SONG; KIM, 2009; BALL, 2007; VENU et al., 2011; OSKUEE et al., 2016, DARROUDI et al., 2014). Diversos micorganismos, entre eles: Staphylococcus aureus e Staphylococcus epidermidis e Candida kruzei, vem adquirindo resitência a vários antimicrobianos (vancomicina, penicilina, oxacilina, miticilina) e antifungos (fluconazol, voriconazol) convencionais (VIEIRA; NASCIMENTO, 2017; BERMAN; KRYSAN, 2020; KERN; RIEG, 2020).

Deste modo, o presente trabalho teve como objetivo sintetizar nanopartículas de prata a partir de mel de abelhas Apis mellifera produzidos na cidade de Santarém-Pará, Brasil. Além disso, foram avaliadas as atividades antimicrobianas da AgNPs frente a microrganismos resistentes (Staphylococcus aureus, Staphylococcus epidermidis e Candida krusei). Por fim, foi avaliada a diferença na síntese das AgNPs e na atividade antimicrobiana em relação ao período de colheita do mel.

\section{Material e Métodos}

\subsection{Local de coleta}

Foram coletadas 16 amostras de mel de abelha Apis melifera, em dois períodos, chuvoso (MC) e seco (MS), sendo oito amostras em cada período, por meio de extração manual sem nenhum utilização de produtos químicos ou fumaça, obtidas em oito apiários localizados nas comunidades Cipoal (M01), Cedro (M02), Bueira (M03), Boa Fé NA (M04), Boa Fé AP (M05), Tipizal (M06), Jacamim (M07) e Terra Amarela (M08), na região de Santarém - Pará, Brasil (02 $35^{\circ}$ '20,78” Sul e $54^{\circ} 41^{\prime} 080^{\prime \prime}$ Oeste á $02^{\circ} 38^{\prime} 26^{\prime \prime}$ Sul e $54^{\circ} 35^{\prime} 47,53^{\prime \prime}$ Oeste).

\subsection{Síntese de Ag-NPs}

A síntese das AgNPs foi realizada utilizando a metodologia empregada por Oskuee et al. (2016). As suspensões coloidais foram preparadas por redução da $\mathrm{Ag}^{+}$por compostos fenólicos e açúcares presentes no mel, com auxílio de ultrassom. $20 \mathrm{~mL}$ de solução de $\mathrm{AgNO}_{3}$ (Sigma-Aldrich) $0,1 \mathrm{~mol} \mathrm{~L}^{-1}$ contendo $20 \%$ de mel in natura (massa/volume) foram expostos à irradiação ultrassônica em ultrassom (ECO-SONICS Q-2850, Thorton) de alta intensidade sob condições ambiente durante $20 \mathrm{~min}$.

\subsection{Espalhamento dinâmico da luz (DLS)}

Em um tubo eppendorf foi preparada uma solução com $100 \mu \mathrm{L}$ de cada amostra juntamente com $2 \mathrm{~mL}$ de água ultrapura, sendo a solução agitada por aproximadamente 2 min. Foram retirados $900 \mu \mathrm{L}$ de cada solução para análise do tamanho (diâmetro hidrodinâmico (DH)), o índice de polidispersividade (PdI) e potencial Zeta (Zetasizer, Malvern). As análises foram feitas a partir de 10 corridas com 3 ciclos de leitura e ângulo de espalhamento de $173^{\circ}$ e temperatura de $25{ }^{\circ} \mathrm{C}$ em um equipamento ZetaSizer Nano ZS 90 (Malvern Instruments, UK).

\subsection{Obtenção dos microrganismos}

Os microrganismos teste utilizados nos ensaios microbiológicos, foram das espécies Staphylococcus aureus (ATCC 25923), Staphylococcus epidermidis (ATCC 12228) $\left(\mathrm{CEFAR}^{\circledR}\right.$, São Paulo, Brazil) e Cândida krusei (Lab01). As linhagens foram mantidas em meio Nutriente $(\mathrm{NM}-3,0 \mathrm{~g}$ de peptona bacteriológica, 5,0 g extrato de carne, 5,0 g NaCl em solução aquosa contendo $1000 \mathrm{~mL}, \mathrm{pH}$ inicial 7,2-7,4) até a preparação das suspensões bacterianas utilizadas nos ensaios.

\subsection{Análise da Concentração Mínima Inibitória (CMI)}

A concentração mínima inibitória foi determinada frente a duas bactérias: Staphylococcus aureus e Staphylococus epidermidis e uma levedura (Cândida krusei). A técnica de diluição do caldo foi utilizada para averiguar a Concentração Mínima Inibitória das soluções da AgNPs de mel (TAVEIRA et al., 2010).

Uma amostra da solução de AgNP não diluída foi adicionada no primeiro tubo e uma diluição em série dessas em água ultrapura foi estabelecida para obter as seguintes concentrações de AgNPs: 10,79; 5,39; 2,70; 1,35; 0,67; 0,34; 0,$17 ; 0,08\left(\mathrm{mg} \mathrm{mL}^{-1}\right)$. Subsequentemente, $100 \mu \mathrm{L}$ de caldo Mueller Hinton (MHB) (Sigma-Aldrich ${ }^{\circledR}$, St. Louis, EUA), 10 $\mu \mathrm{L}$ de suspensão com $10^{6} \mathrm{UFC} \mathrm{mL}^{-1}$ e $100 \mu \mathrm{L}$ de cada solução de AgNP foram adicionado a cada poço para CMI (KHAN et al., 2014). $10 \mu \mathrm{L}$ de solução de cloreto de 2,3,5-trifenil tetrazóico (CTT) $(0,01 \%)$ foi usada como corante químico. $\mathrm{O}$ controle negativo foi realizado substituindo as amostras AgNPs por caldo de Mueller Hinton (MHB) e controle positivo foi realizada substituindo amostras de AgNPs por 100 
$\mu \mathrm{L}$ de antibiótico ampicilina $\left(100 \mu \mathrm{g} \mathrm{mL}^{-1}\right)$. Todas as placas foram incubadas a $35^{\circ} \mathrm{C}$ por $24 \mathrm{~h}$.

\subsection{Análises estatísticas}

Os dados quantitativos dos experimentos, realizados em triplicata, foram apresentados em média \pm desvio padrão.

\section{Resultados e Discussão}

\subsection{Diâmetro hidrodinâmico e potencial Zeta}

Todas as AgNPS sintetizadas a partir do mel do período chuvoso (MC) e do período seco (MS) obtiveram diâmetro hidrodinâmico médio de $600 \mathrm{~nm}$ (Quadro 1). Oskuee et al. (2016), que realizou o mesmo processo de síntese, obteve o tamanho de partícula variando entre 50 e $250 \mathrm{~nm}$. El-desouky et al. (2016) ao sintetizar AgNP a partir do mel observou o tamanho médio de 9,9 $\mathrm{nm}$. Hosny et al (2017) verificou nanopartículas com tamanho predominante de 4,2 nm, sendo que as amostras mais ácidas apresentaram valor predominante de $11,7 \mathrm{~nm}$.

Quadro 1 - Tamanhos e valores das polidispersões das AgNPs sintetizadas

\begin{tabular}{|c|c|c|c|c|c|}
\hline Amostras & DH (nm) & PdI & Amostras & DH (nm) & PdI \\
\hline MC01 & $693 \pm 45,9$ & $0,33 \pm 0,02$ & MS01 & $552 \pm 33,3$ & $0,33 \pm 0,01$ \\
\hline MC02 & $586 \pm 8,3$ & $0,31 \pm 0,06$ & MS02 & $468 \pm 34,4$ & $0,47 \pm 0,09$ \\
\hline MC03 & $620 \pm 36,6$ & $0,23 \pm 0,03$ & MS03 & $792 \pm 51,4$ & $0,29 \pm 0,03$ \\
\hline MC04 & $739 \pm 60,7$ & $0,30 \pm 0,09$ & MS04 & $461 \pm 12,4$ & $0,43 \pm 0,13$ \\
\hline MC05 & $637 \pm 27,2$ & $0,27 \pm 0,04$ & MS05 & $445 \pm 35,9$ & $0,32 \pm 0,04$ \\
\hline MC06 & $557 \pm 3,9$ & $0,26 \pm 0,01$ & MS06 & $698 \pm 24,1$ & $0,35 \pm 0,04$ \\
\hline MC07 & $619 \pm 28,6$ & $0,27 \pm 0,02$ & MS07 & $692 \pm 30,5$ & $0,33 \pm 0,02$ \\
\hline MC08 & $748 \pm 47,9$ & $0,28 \pm 0,02$ & MS08 & $823 \pm 41,9$ & $0,35 \pm 0,09$ \\
\hline Am0stras & \multicolumn{2}{|c|}{ Potencial zeta } & Amostras & Potencial zeta \\
\hline MC01 & $2,39 \pm 0,14$ & MS01 & $5,07 \pm 0,33$ \\
\hline MC02 & $3,07 \pm 0,17$ & MS03 & $-1,96 \pm 0,27$ \\
\hline MC03 & $5,84 \pm 0,17$ & MS04 & $1,84 \pm 0,10$ \\
\hline MC04 & $4,98 \pm 0,33$ & MS06 & $-2,64 \pm 0,37$ \\
\hline MC05 & $5,35 \pm 0,34$ & MS07 & $-2,35 \pm 0,38$ \\
\hline MC06 & $6,62 \pm 0,09$ & $-0,39 \pm 0,14$ \\
\hline MC07 & $4,62 \pm 0,28$ & $-1,31 \pm 0,30$ \\
\hline
\end{tabular}

Fonte: Dados da pesquisa.

Os valores encontrados na literatura são bem discrepantes quando comparado neste trabalho, isso pode está relacionado principalmente processo de síntese, armazenamento, $\mathrm{pH}$, a concentração do precursor, a concentração do redutor, o tempo de incubação ou outras variáveis ligada a $\mathrm{AgNO}_{3}$ ou relacionado ao peso molecular dos componentes do mel (UMOREN et al., 2014; HOSNY et al., 2017). Vale destacar que Panáček (2006) sintetizou AgNPs a partir de açúcares e obteve tamanhos maiores que $100 \mathrm{~nm}$.

$\mathrm{O}$ índice de polidispersividade (PdI) médio para as AgNps de méis do período seco e chuvoso foi de 0,36 e 0,28, respectivamente. Isso mostra que as AgNPs não tendem a ficar aglomeradas, ou seja, são homogêneas (Tabela 1). Esse é um fator importante, pois desse modo não há perda de atividade e assim de possíveis aplicações.

O potencial Zeta é a medição da magnitude da repulsão de carga eletrostática ou atração entre partículas em suspensão líquida. Ele é um dos essenciais parâmetros para caracterizar a estabilidade de nanopartículas em ambiente aquoso. As nanopartículas que apresentam o potencial Zeta maior que $\pm 30 \mathrm{mV}$ possuem uma excelente estabilidade (MARCATO, 2009). O potencial Zeta foi negativo para as amostras MSL
2, 4, 5, 6 e 7 do período seco. Umoren et al. (2014) sugere que potencial negativo mostrado pelas AgNPs pode ser devido ao possível revestimento dos componentes bio-orgânicos presentes no mel.

\subsection{Concentração Mínima Inibitória - CMI}

As amostras de nanopartículas foram analisadas para a determinação da CMI frente aos microrganismos teste sensíveis ao produto. Todas as amostras apresentaram atividade antimicrobiana frente aos microrganismos testados (Quadro 2).

Quadro 2 - Atividade antimicrobiana (CMI) das amostras de mel de abelha

\begin{tabular}{|c|c|c|c|}
\hline \multirow{2}{*}{ Amostras } & \multicolumn{3}{|c|}{ Concentração Mínima Inibitória (mg mL $\left.\mathbf{~}^{-1}\right)^{*}$} \\
\cline { 2 - 4 } & S. aureus & S. epidermidis & C. kruzei \\
\hline MS01 & 10,79 & 10,79 & 0,67 \\
\hline MS02 & 10,79 & 10,79 & 0,67 \\
\hline MS03 & 0,17 & 0,17 & 0,08 \\
\hline MS04 & 0,17 & 0,17 & 0,08 \\
\hline MS05 & 10,79 & 10,79 & 0,67 \\
\hline MS06 & 10,79 & 10,79 & 0,67 \\
\hline MS07 & 10,79 & 10,79 & 0,67 \\
\hline MS08 & 10,79 & 10,79 & 0,67 \\
\hline
\end{tabular}




\begin{tabular}{|c|c|c|c|}
\hline MC01 & 0,67 & 0,67 & 10,79 \\
\hline $\mathrm{MCO} 2$ & 0,67 & 0,67 & 10,79 \\
\hline $\mathrm{MC03}$ & 0,67 & 0,67 & 10,79 \\
\hline $\mathrm{MC} 04$ & 0,67 & 0,67 & 10,79 \\
\hline MC05 & 0,67 & 0,67 & 10,79 \\
\hline $\mathrm{MC06}$ & 0,67 & 0,67 & 10,79 \\
\hline MC07 & 0,67 & 0,67 & 10,79 \\
\hline MC08 & 0,67 & 0,67 & 10,79 \\
\hline Ampicilina* & 100 & 100 & 100 \\
\hline $\mathrm{AgNO}_{3}$ & 10,79 & 10,79 & 5,39 \\
\hline
\end{tabular}

Fonte: Dados da pesquisa.

A concentração mínima inibitória obtida para $S$. aureus e S. epidermides foi de $10,79 \mathrm{mg} \mathrm{mL}^{-1}$ nas amostras do período seco, exceto MS 3 e 4 que teve o CMI de $0,17 \mathrm{mg} \mathrm{mL}^{-1}$. Já as do período chuvoso apresentou o CMI de $0,67 \mathrm{mg} \mathrm{mL}^{-1}$. C. krusei apresentou CMI de $0,67 \mathrm{mg} \mathrm{mL}^{-1}$ nas amostras do período seco, exceto MS 3 e $4\left(0,08 \mathrm{mg} \mathrm{mL}^{-1}\right)$, no período chuvoso o CMI foi de $10,79 \mathrm{mg} \mathrm{mL}^{-1}$. A solução de $\mathrm{AgNO}_{3}$ inibiu o crescimento microbiano de todos os microrganismos testado, S. epidermidis e S. aureus com CMI de $10,79 \mathrm{mg} \mathrm{mL}^{-1}$ de Ag.

Sreelakshmi et al. (2011) e Hosny (2017) encontraram valores de 6,25 e $2,81 \mu \mathrm{g} \mathrm{mL} \mathrm{m}^{-1}$, respectivamente, para Staphylococcu aureus. Já Oskuee et al. (2016) encontrou o valor de CMI de $19,5 \mu \mathrm{g} \mathrm{mL}^{-1}$ para E. coli $\mathrm{e} S$. aureus. A menor ação antimicrobiana das AgNPs sintetizadas neste trabalho uma vez que a ação antimicrobiana dessas nanopartículas obtidas a partir de sintese verde é bastante dependente do tamanho, concentração e carga superficial (GOPINATH et al., 2013; FRANCI et al., 2015). As AgNPs com menores tamanhos possuem maiores áreas superficiais em relação ao volume e, por meio de atração eletrostática entre a superfície celular carregada negativamente e a carga positiva dos íon $\mathrm{Ag}^{+}$, contribuindo para a rápida absorção e elevada atividade antimicrobiana (SHANMUGAM; BHARATH, 2017; KUMAR et al., 2018; HAMOUDA et al., 2019). No entanto, as nanoparticulas com tamanhos maiores, como as obtidas neste trabalho não são capazes de entrar nas células microbianas, o que diminui a sua ação antimicrobiana.

De acordo com estudos as AgNPs sintetizadas a base de mel se mostram mais ativas em bactérias Gram negativas, onde a parede celular mais espessa do Gram positivo permite menor entrada de prata na membrana citoplasmática do que a Gram negativa.

\section{Conclusão}

Foram biossintetizadas AgNPs a partir dos diversos méis produzidos na região de Santarém-PA em períodos distintos de sazonalidade. O elevado tamanho das AgNPs combinado com uma polidispersão média em solução aquosa foram observados por análises de DLS e pode ter influenciado nos valores de CMI. Bactérias Gram-positivas foram mais sensíveis às AgNPs produzidas pelos méis coletados no período chuvoso e pelas amostras MS03 e MS04 do período seco. Do contrário, os valores de CMI foram menores para a levedura quando as AgNPs foram produzidas a partir dos méis do período seco, com destaque para as amostras MS03 e MS04 com 0,08 $\mathrm{mg} \mathrm{mL}^{-1}$. Dessa forma, conclui-se que o mel é um bom agente redutor na formação de AgNPs biogênicas e sua exploração é bastante relevante devido suas características intrínsecas e agregação de valor junto à nanobiotecnologia.

\section{Agradecimentos}

Projeto 88881.149143/2017-01, financiado pela Coordenação de Aperfeiçoamento de Pessoal de Nível Superior (CAPES) e Fundação Paraense de Amparo à Pesquisa (FAPESPA). Os autores também agradecem aos apicultores do oeste do Pará, Brasil.

\section{Referências}

BALASOORIYA, E.R. et al. Honey mediated green synthesis of nanoparticles: new era of safe nanotechnology. J. Nanomaterials, v.201, 2017. doi:10.1155/2017/5919836.

BALL, D.W. The chemical composition of honey. J. Chem. Education, v.84, n.10, p.1643-1646, 2007. doi:10.1021/ ed084p1643

BANDEIRA, A.M.P. et al. Antioxidant activity and physicochemical characteristics of honeys from the eastern Amazon region, Brazil. Acta Amazonica, v.48, n.2, p.158-167, 2018. doi: $10.1590 / 1809-4392201702721$

BERMAN, J.; KRYSAN, D.J. Drug resistance and tolerance in fungi. Nat. Rev. Microbiol., v.18, p.319-331, 2020. doi: 10.1038/ s41579-019-0322-2

DARROUDI, M. et al. Food-directed synthesis of cerium oxide nanoparticles and their neurotoxicity effects. Ceram. Int., v.40, n.5, p.7425-7430, 2014. doi: 10.1016/j.ceramint.2013.12.089

EL-DESOUKY, T.A.; AMMAR, H.A.M. Honey mediated silver nanoparticles and their inhibitory effect on aflatoxins and ochratoxin A. J. Appl. Pharm. Sci., v.6, n.6, p.83-90, 2016. doi: 10.7324/JAPS.2016.60615

FRANCI, G. et al. Silver nanoparticles as potential antibacterial agents. Molecules, v.20, n.5, p.8856-8874, 2015. doi: $10.3390 \% 2$ Fmolecules 20058856

GOPINATH, V. et al. Biogenic synthesis of antibacterial silver chloride nanoparticle using leaf extracts of Cissus quadrangularis Linn. Mater Lett., v.91, p.224-227, 2013. doi: 10.1016/j. matlet.2012.09.102

GONZÁLEZ FÁ, A.J.; JUAN, A.; DI NEZIO, M.S. Synthesis and characterization of silver nanoparticles prepared with honey: the role of carbohydrates. Anal. Lett., v.50, n.5, p.877-888, 2017. doi: 10.1080/00032719.2016.1199558

HAMOUDA, R.A. et al. Synthesis and biological characterization of silver nanoparticles derived from the cyanobacterium Oscillatoria limnetica. Sci. Rep., v.9, n.13071, 2019. doi: 10.1038/s41598-019-49444-y

HOSNY, A.M.S. et al. Antimicrobial activity of silver nanoparticles synthesized using honey and gamma radiation against silver-resistant bacteria from wounds and burns. $A d v$. Nat. Sci.: Nanosci. Nanotechnol., v.8, n.4, p.045009, 2017. doi: 10.1088/2043-6254/aa8b44

KERN, W.V.; RIEG, S. Burden of bacterial bloodstream infection: 
a brief update on epidemiology and significance of multidrugresistant pathogens. Clin. Microbiol. Infect., v.26, n.2, p.151-157, 2020. doi: 10.1016/j.cmi.2019.10.031

KHAN, F. et al. Antimicrobial properties and isotope investigations of South African honey. J.App. Microbiol., v.117, n.2, p.366-379, 2014. doi: 10.1111/jam.12533

KUMAR, S.S.D. et al. Cellular imaging and bactericidal silver mechanism of green-synthesized solver nanoparticle against human pathogenic bacteria. J. Photochem. Photobiol. B., v.178, p.259-269, 2018. doi: 10.1016/j.jphotobiol.2017.11.001

LAVINAS, F.C. et al. Brazilian stingless bee propolis and geopropolis: promising sources of biologically active compounds. Rev. Bras. Farmacogn., v.29, n.3, p.389-399. doi: 10.1016/j. bjp.2018.11.007

MARCATO, P.D.D. Preparação, caracterização e aplicações em fármacos e cosméticos de nanopartículas lipídicas sólidas. Rev. Eletr. Farm., v.6, n.2, p.1-37, 2009. doi: 10.5216/ref.v6i2.6545

MEIRELES, S.; CANÇADO, I.A.C. Mel: parâmetros de qualidade e suas implicações para a saúde. Synthesis Revista Digital FAPAM, v.4, n.4, p.207-219, 2016.

OSKUEE, R.K. et al. Honey-Based and Ultrasonic-Assisted Synthesis of Silver Nanoparticles and Their Antibacterial Activities. J. Nanosc. Nanotechnol., v.16, n.8, p.7989-7993, 2016. doi: 10.1166/jnn.2016.13031

PANÁC̆EK, A. et al. Silver colloid nanoparticle: synthesis, characterization and their antibacterial activity. J. Phys. Chem. B, v.110, n.33, p.16248-16253, 2006. doi: 10.1021/jp063826h

RAI, M.; YADAV, A.; GADE, A. Silver nanoparticles as a new generation of antimicrobials. Biotechnol. Adv., v.27, n.1, p.76-83, 2009. doi: 10.1016/j.biotechadv.2008.09.002
RAI, M. Nanobiotecnologia verde: biossínteses de nanopartículas metálicas e suas aplicações como nanoantimicrobianos. Cienc. Cult., v.65, n.3, p.44-48, 2013. doi: 10.21800/S000967252013000300014

SONG, J.Y.; KIM, B.S. Rapid biological synthesis of silver nanoparticles using plant leaf extracts. Bioprocess Biosyst. Eng., v.32, n.1, p.79-84, 2009. doi: 10.1007/s00449-008-0224-6

SREELAKSHMI, C. et al. Honey derivatized $\mathrm{Au}$ and $\mathrm{Ag}$ nanoparticles and evaluation of its antimicrobial activity. $J$. Nanosci. Nanotechnol., v.11, n.8, p.6995-7000, 2011. doi: 10.1166/jnn.2011.4240

SHANMUGAM, R.; BHARATH, L.V. Mechanism of plantmediated synthesis of silver nanoparticles: a review on biomolecules involved, characterisation and antibacterial activity. Chem.-Biol. Interact., v.273, p.219-227. doi: 10.1016/j. cbi.2017.06.019

TAVEIRA, M. et al. Lycopersicon esculentum Seeds: An Industrial Byproduct as an Antimicrobial Agent. J. Agric. Food Chem., v.58, n.17, p.9529-9536, 2010. doi: 10.1021/jf102215g

UMOREN, S.A.; OBOT, I.B.; GASEM, Z.M. Green synthesis and characterization of silver nanoparticles using red apple (Malus domestica) fruit extract at room temperature. J. Mater. Environ. Sci., v.5, n.3, p.907-914, 2014.

VENU, R. et al. Bio-directed synthesis of platinum nanoparticles using aqueous honey solutions and their catalytic applications. Colloids Surf. A Physicochem. Eng. Asp., v.384, n.1-3, p.733-738, 2011. doi: 10.1016/j.colsurfa.2011.05.045

VIEIRA, F.; NASCIMENTO, T. Candida antifungal resistance and therapeutic approach. Rev. Port. Farmacoter., v.9, p.161-168, 2017. 